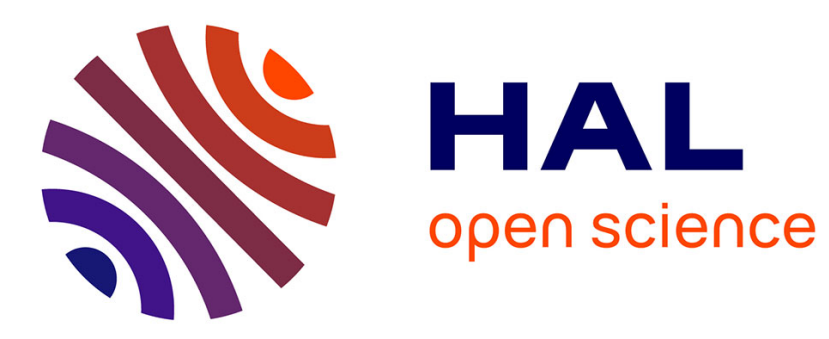

\title{
Ultrafast turbidity compensation in the optical therapeutic window by three-wave mixing phase conjugation
}

Mischa Guillerm, Fabrice Devaux, Luc Froehly, Luca Furfaro, Éric Lantz

\section{To cite this version:}

Mischa Guillerm, Fabrice Devaux, Luc Froehly, Luca Furfaro, Éric Lantz. Ultrafast turbidity compensation in the optical therapeutic window by three-wave mixing phase conjugation. Journal of Optics, 2013, 15, pp.055204. 10.1088/2040-8978/15/5/055204 . hal-00879030

\section{HAL Id: hal-00879030 \\ https://hal.science/hal-00879030}

Submitted on 31 Oct 2013

HAL is a multi-disciplinary open access archive for the deposit and dissemination of scientific research documents, whether they are published or not. The documents may come from teaching and research institutions in France or abroad, or from public or private research centers.
L'archive ouverte pluridisciplinaire HAL, est destinée au dépôt et à la diffusion de documents scientifiques de niveau recherche, publiés ou non, émanant des établissements d'enseignement et de recherche français ou étrangers, des laboratoires publics ou privés. 


\title{
Ultrafast turbidity compensation in the optical therapeutic window by three-wave mixing phase conjugation
}

\author{
Mischa Guillerm, Fabrice Devaux, Luc Froehly, Luca Furfaro \\ and Eric Lantz \\ Institut FEMTO-ST, Département d'Optique P. M. Duffieux, \\ UMR 6174 CNRS / Université de Franche-Comté - 16 route de Gray, 25030 Besancon \\ - France \\ E-mail: eric.lantz@univ-fcomte.fr
}

\begin{abstract}
Imaging by phase conjugation through diffusing media is performed by using parametric amplification in a type II crystal, at a wavelength included in the therapeutic window. By nature, the method ensures imaging in a time far below the decorrelation time of in vivo biological tissues. A systematic comparison of performances with direct imaging is provided.
\end{abstract}

\section{Introduction}

At present, one of the most mature technique for imaging in biological media is optical coherence tomography [1], where images are reconstructed slice-by-slice from the optical interference of coherent reflection from the biological medium with a known reference beam. However, in the optical therapeutic window where light absorption is weak, i.e in the red to near-infrared range, such a coherent method is limited to depths smaller than two millimeters, because for greater depths all light is scattered and no interferometric signal remains for image reconstruction. For thicker tissues, various methods have been developed that use the scattered light, like diffuse imaging i.e. reconstruction by computation from diffused waves [2], time gating to isolate the less diffused photons [3], optoacoustic methods [4] and imaging by two-photon absorbtion [5].

Fortunately, in static media scattering is a deterministic phenomenon that can be, at least in principle, reversed. It was demonstrated 40 years ago [6] that a wave scattered by a ground glass slide can be recovered and restored to its initial state by applying optical phase conjugation and back propagation through the same slide. A recent experiment by Yaqoob et al showed that such a scheme can also be applied to a biological medium [7], though not in vivo. An image transmitted through chicken breast was recorded as a hologram in a photorefractive crystal, and then restored by back propagation of the phase-conjugated wave through the same biological medium. Since the position of the scatterers did not change between forward and backward propagation, 
the image resolution was preserved. Because of the strong absorption of the $532 \mathrm{~nm}$ wavelength used in the experiments, the thickness was limited to $0.7 \mathrm{~mm}$, and the acquisition time in the minute range did not allow studying in vivo biological media, which typically have a decorrelation time of $0.1 \mathrm{~ms}$ [8]. In a later experiment [9] a collimated beam (no image) was reconstructed, with a hologram recording time reduced to 20 seconds and an increase to $7 \mathrm{~mm}$ of the thickness of the chicken breast.

Nonlinear $\chi^{2}$ imaging by three-wave mixing allows phase-conjugation in nanoseconds of a near-infrared image. We showed several years ago that an image distorted by an aberrant plate can be restored [10]. This method is one of the numerous methods that allows the ultrafast suppression of image distortions by phase conjugation, for example through a clear plastic using a hybrid amorphous silicon/ferroelectricliquid-crystal device [12], through turbulent atmosphere using sodium vapor [13], or through textured plastic sheets using stimulated Brillouin scattering [14]. In all these experiments, the distorting medium was transparent and our own experiments with such type of medium (aberrant glass plate, adhesive ribbon) showed that depolarization is negligible even in situations where direct imaging is impossible. We propose in this paper to use phase-conjugation by three-wave mixing, like in ref. [10], for imaging through a diffusing medium of latex microbeads. Because the polarization is not conserved during the traversal of the medium, unlike for an aberrant plate, this new experiment is designed to ensure the same polarization for the forward and the backward wave.

\section{Experiment principle and setup}

In parametric amplification, the idler is generated with wave-vectors opposite to the signal for the two transversal dimensions, giving forward phase conjugation [15]. In this work, like in [10], reflection of the idler wave on the coated exit face of the crystal reverses the idler wave vector along the mean propagation direction and leads to full phase conjugation (see fig.1). A pulsed $1064 \mathrm{~nm}$ beam illuminates an USAF resolution chart, passes through the scattering medium and is phase-conjugated in a Potassium Titanyl Phosphate (KTP) crystal by three-wave mixing with an intense $532 \mathrm{~nm}$ pump pulse. This crystal is well known for its high nonlinear coefficient and its particularly favorable phase-matching properties for this couple of wavelengths [16]. The phase-conjugated wave returns through the scattering medium and is imaged on a CCD camera.

The coated crystal face reflects both the signal and the idler. In a type II crystal, the signal and the phase-conjugated wave have orthogonal polarizations, suggesting the use of a polarizer to reject the signal. However, unlike in reference [10] where aberrations were more or less polarization independent, scattering depends strongly of polarization: as an example, we measured the degree of polarization after the traversal of the tank filled with microbeads as in fig.3c and found a ratio of 3.6 between the powers on the initial polarization and on the orthogonal one. The value of this ratio is 500 for a tank filled with water. Hence, we wish to keep identical the polarization of the waves exiting from the sample and returning inside it. This is done by combining a polarizer with 


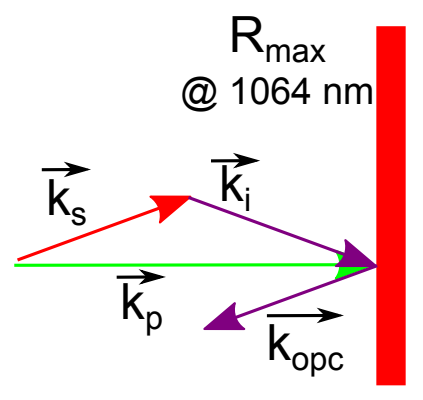

Figure 1. Phase-conjugation by parametric amplification: $k_{s}, k_{i}, k_{p}$ and $k_{o p c}$ are respectively the signal, idler, pump and phase-conjugated wave vectors.

a Faraday rotator: the signal is first linearly polarized at the output of the diffusing medium, then its polarization is rotated by a $45^{\circ}$ angle. The generated phase-conjugated wave has a polarization orthogonal to the signal, and both return through the rotator. Their polarization turns again by $45^{\circ}$, in the same direction as before. On the whole, the signal polarization turns by $90^{\circ}$ and is therefore rejected by the polarizer, while the phase-conjugated wave is transmitted. This reasoning is strictly valid only for a plane wave propagating along the optical axis of the rotator. However, the part of the field that traverses the isolator and returns phase-conjugated can be considered as a superposition of plane waves with wavevectors contained in the cone allowed by phasematching. For a plane wave whose wavevector makes an angle $\theta$ with the optical axis of the rotator, the rotation of polarization is proportional to $\cos (\theta)$ [11], ensuring a negligible difference with the collinear case for the small angles contained in the phase matching cone.

The experimental setup is depicted in fig.2. The infrared beam at $1.064 \mu \mathrm{m}$ is

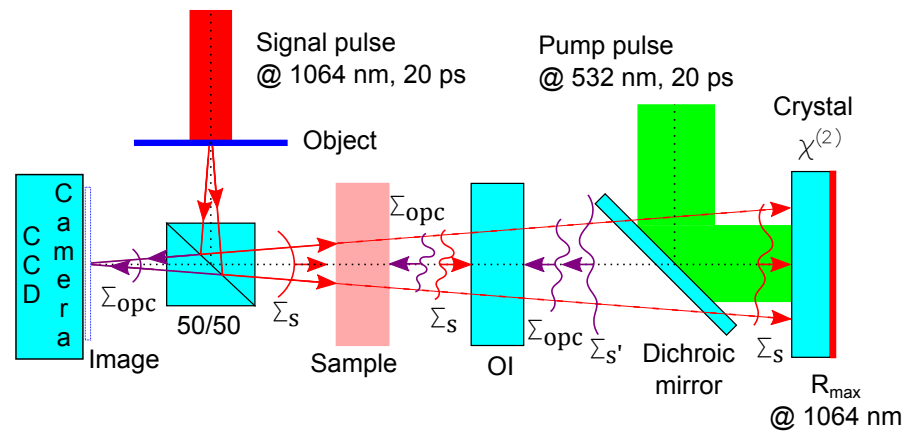

Figure 2. Experimental setup.

delivered by a Q-switched mode-locked $N d: Y A G$ laser (from Quantel). The nearly $T E M_{00}$ infrared pulses have a 55 ps duration (FWHM), a time-bandwidth product close to 1 and a $10 \mathrm{~Hz}$ repetition rate. The radiation is partially frequency-doubled in a Potassium Dihydrogen Phosphate (KDP) crystal. The remaining infrared light is separated from the green light by a dichroic mirror and then attenuated and vertically polarized. The infra-red pulse illuminates an USAF resolution target, then is reflected 
on a 50/50 intensity beam-splitter and illuminates the diffusing medium. The exit wave $\Sigma_{s}$ traverses an optical isolator (OI), is superimposed with the green pulse at the dichroic mirror and interacts with the pump in a $5 \times 5 \mathrm{~mm}^{2}$ wide and $2.5 \mathrm{~mm}$ long KTP crystal (from Cristal laser SA). Both the signal $\Sigma_{s^{\prime}}$ and phase-conjugated wave $\Sigma_{o p c}$ backpropagate towards the isolator, where the signal is rejected while the phase-conjugated wave traverses back the diffusing medium. After transmission by the beam-splitter, the image formed by the phase-conjugated wave is recorded on a scientific CCD camera (Princeton Instruments, Spec-10 400B), at a position symmetrical of the object. The distances between the diffusing medium and either the object or the crystal are both equal to $20 \mathrm{~cm}$. The signal gain $G_{s}$, defined as the ratio between the signal powers with and without pump, has been measured with a power meter, giving a value close to 2. Because the added power on the signal is, for identical signal-idler wavelengths, equal to the generated idler power, the gain of phase conjugation $G_{i}$, defined as the ratio between the incident signal power and the phase conjugated power, is given by $G_{i}=\left(G_{s}-1\right)$, because of the coatings ensuring at $1064 \mathrm{~nm}$ a total reflection at the rear face of the crystal and a total transmission at the front face. While the object is not imaged on a plane close or inside the diffusing medium in fig.2, we have performed a second experiment where an additional lens produces an intermediate image at the input plane of the diffusing medium. For direct imaging, this "curtain effect" configuration is known to be optimal [17]. Using pulses allows the study of moving samples, such as a suspension of microbeads, as the the medium does not need to be static from one pulse to another, but only for the time the light takes to travel from the sample to the KTP crystal and back. In our experiment, this duration is of the order of $1 \mathrm{~ns}$, small enough to consider the samples as static [8]. On the other hand, we can extend the acquisition time to include more than one pulse for one image. In practice we used 10 pulses to make an acquisition.

\section{Imaging through thick scattering media}

\subsection{Phase-conjugation experiments}

A transparent tank with a $1 \mathrm{~cm}^{2}$ square base was filled with $2 \mathrm{~mL}$ of deionized water. Latex microbeads of $1.1 \mu \mathrm{m}$ diameter from a mother solution (supplied by SigmaAldrich) were successively added in $2 \mu \mathrm{L}$ quantities. The scattering coefficient of the mother solution was computed equal to approximately $1600 \mathrm{~cm}^{-1}$. Latex microbeads are commonly used scatterers phantoms and their optical properties can be computed with Mie theory. The fifth element of the zeroth group of an USAF resolution test chart was chosen as the object, corresponding to roughly $2 \mathrm{lp} / \mathrm{mm}$ resolution. The first two images of fig. 3 were made through water, with respectively the phase-conjugated and the signal wave (in this latter case, the isolator is removed and the pump is turned off). All throughout the paper, the lateral size of all the square images is $2 \mathrm{~mm}$, corresponding on the camera to 100 pixels of $20 \times 20 \mu \mathrm{m}^{2}$. The third figure depicts the last image where 
the object can be recognized and corresponds to a concentration of $0.6 \%$. To quantify the quality of this image, we have computed the normalized intercorrelation function between this image and the phase-conjugated image through water. The maximum of this function is 0.77 while the maximum intercorrelation of the image through water (fig.3a) with the signal (fig.3b) is 0.47 . Note we have employed a Fourier transform algorithm to calculate this correlation, in order to obtain a result insensitive to a shift of one image with respect to the other. This intercorrelation coefficient can be also interpreted, with caution, as an estimate of the image-to-background ratio [18]: see Appendix. From Mie theory we derived the optical properties of the sample: $\mathrm{mfps}=0.10$ $\mathrm{cm}, \mathrm{mfps}^{\prime}=0.81 \mathrm{~cm}, \mathrm{mfpa}=10.9 \mathrm{~cm}$ and $\mathrm{g}=0.87$ are respectively the scattering, reduced scattering, absorption Mean Free Paths and anisotropy parameter. As the tank is 1 $\mathrm{cm}$ thick, there are on average 10 scattering, 1.23 reduced scattering and less than 1 absorption events. When the object is imaged at the input face of the diffusing medium (curtain effect configuration), no improvement is obtained: the last visible image corresponds in this case to 8 mean free paths. We have repeated the experiment by removing the isolator and replacing it by a polarizer in front of the camera, in the direction corresponding to the idler if no depolarization occurs in the diffusing medium. Results are worse : the last concentration where the image is recognizable is $0.3 \%$. This result is in agreement with our measurement of a quite important depolarization ratio (see above).

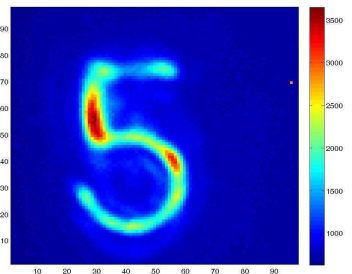

(a)

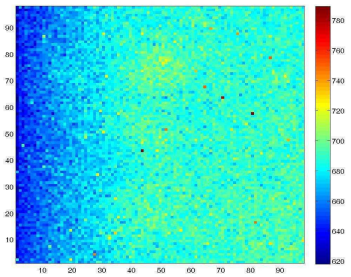

(b)

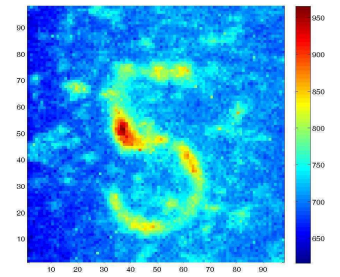

(c)

Figure 3. (a) Phase-conjugated image through water, (b) signal image, without pump and isolator, through water, (c) phase-conjugated image through $12 \mu \mathrm{L}$ of microbeads.

\subsection{Single-pass experiment}

The phase conjugation performances were compared to single-pass conventional imaging through the same medium. The isolator, dichroic mirror and KTP crystal were removed and a lens $\mathrm{L}$ was added to make an image on the camera, as depicted in fig. 4. The distances between the lens and either the object or the image are both equal to $20 \mathrm{~cm}$, i.e. twice the focal length of the lens. The curtain effect configuration was also tested by adding a second lens.

The same object was used and again the microbeads were added progressively, images were acquired until the object could no longer be resolved. The last resolved image was obtained for a $1.1 \%$ concentration, corresponding to $\mathrm{mfps}=0.06 \mathrm{~cm}$, 


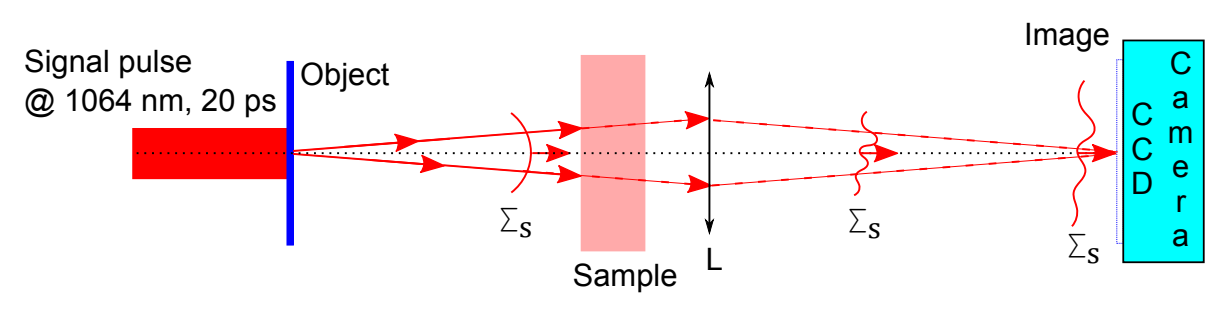

Figure 4. Single-pass experiment.

mfps' $=0.45 \mathrm{~cm}, \mathrm{mfpa}=6.0 \mathrm{~cm}$ and $\mathrm{g}=0.87$. The tank size was still $1 \mathrm{~cm}$, corresponding on average to 16.7 scattering (17.6 in the curtain effect configuration), 2 reduced scattering and less than 1 absorption events.

\subsection{Analysis}

Considering the length of the tank, $1 \mathrm{~cm}$, the absorption was negligible in both experiments. The anisotropy parameter was concentration independent and therefore constant; its value close to 1 indicates a favored forward scattering direction. In the single-pass experiment, the 16 scattering events correspond to less than the 20 scattering events experienced by the light after a round trip in the phase conjugation scheme but more than the 10 scattering events corresponding to a single-pass in this configuration. In other words, we have demonstrated that phase conjugation allows an improvement as compared to the diffusion corresponding to a round trip. However, this improvement is not sufficient to lead to results better than in a single-pass configuration.

\section{Phase-conjugation fidelity}

\subsection{Fraction of power intercepted by the crystal}

The most evident limitation of parametric phase conjugation is related to the fraction of the etendue of the scattered wave that can be phase-conjugated in the crystal. Despite the high anisotropy parameter of the diffusing medium, the lateral size of the diffused spot becomes at the exit of the cell much larger than that of the KTP crystal and only a small fraction of the power can enter the crystal after passing the isolator, with a small divergence ensuring no limitation due to phase matching. This situation corresponds to partial phase-conjugation as described by $\mathrm{Gu}$ and Yeh in reference [18]. A real optical phase-conjugating mirror, such as our KTP crystal, can be modeled as a perfect phase-conjugate mirror of infinite extent in series with an aperture. The reflected wave is made of two parts: a phase-conjugated component, which faithfully reproduces the original image, and a non-phase conjugate component which acts as noise. Assuming no back-scattered radiation and no absorption, $\mathrm{Gu}$ and Yeh showed that the image-tobackground ratio I/B for partial conjugation is given :

$$
\frac{I}{B}=\frac{|\alpha|}{1-|\alpha|}
$$


where $|\alpha|$, is equal to the fraction of power intercepted by the phase-conjugate mirror:

$$
|\alpha|=\frac{a}{A} \text {. }
$$

where $a$ is the surface of the phase-conjugate mirror, the transverse section of our crystal in our case by assuming a larger pump transverse section, and $A$ is the area of the scattered spot after a virtual propagation until the input plane of the crystal without the limitations introduced by the optical elements : see fig.5.

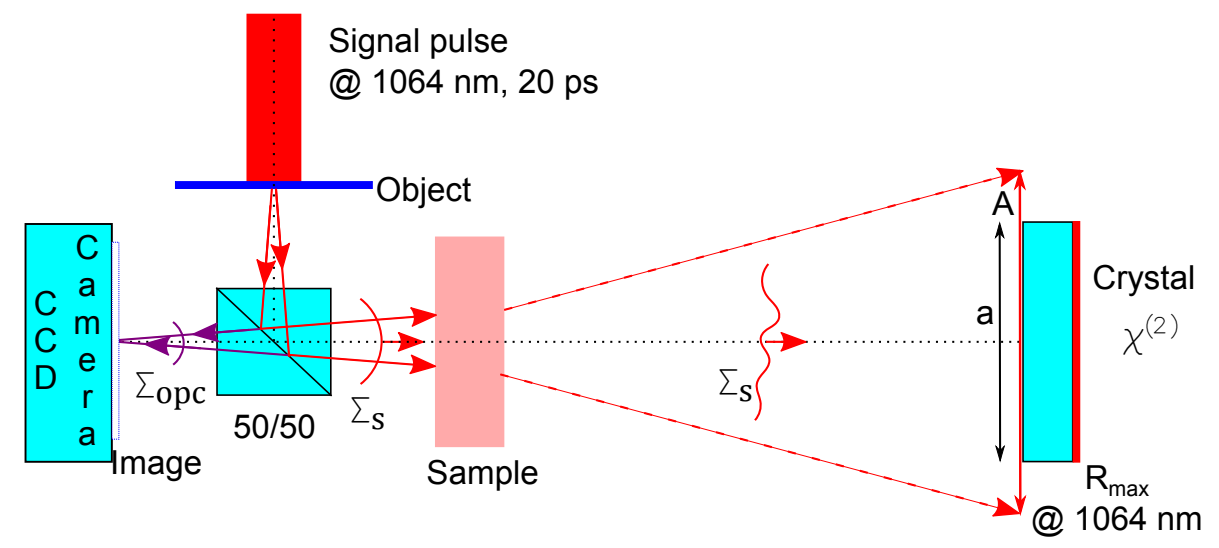

Figure 5. Fraction of power intercepted by the crystal

\subsection{Etendue corresponding to phase conjugation}

The above analysis is correct inasmuch as the beam has a small divergence at the input of the phase-conjugate mirror, ensuring a quasi-perfect phase conjugation of this beam. Though this divergence is smaller than the phase-matching range in our experiment, because of pupils before the crystal, we prefer to conduct the analysis by introducing a more fundamental limitation, related to the etendue $e$ that corresponds to phaseconjugation by the crystal, i.e. the product of its illuminated transverse area $a$ by the solid angle $\Omega$ limited either by phase-matching or by the pupils. The theory of Gu and Yeh can be easily generalized to non perfect conjugating mirrors by replacing the areas $a$ and $A$ by the corresponding etendues $e$ and $E$, where $E$ is the etendue of the beam at the exit of the diffusing medium, assuming in first approximation that all the diffused light attains this exit face. After the scattering medium, $\mathrm{E}$ is equal to the product of the output face of the tank, $1 \mathrm{~cm}^{2}$ by the solid angle $\Omega^{\prime}=2 \pi$ under which the diffused light exits the tank, giving an etendue after the scattering medium $E \sim 6.3 \mathrm{~cm}^{2}$.sr. The calculations for the KTP follow the same lines, with the solid angle $\Omega$ corresponding to the wavevectors entering the crystal. This angle, $\Omega=\pi(12 \mathrm{mrad})^{2}$, is limited by the KTP crystal pupil and not by phase matching, giving an etendue:

$$
e \sim 1.1 \times 10^{-4} \mathrm{~cm}^{2} s r \sim \frac{E}{1.5 \times 10^{4}}
$$

This extremely weak value explains the low obtained signal to noise ratio. A more compact experiment would not greatly change this ratio for a thick diffusing medium 
because of the limit due to phase matching. However, the size of the beam at the exit of a thinner medium is smaller, allowing magnification before the crystal and a more favorable ratio between etendues, as is shown in the next section.

\section{Imaging through thin scattering media}

\subsection{Phase-conjugation experiment}

The etendue of the beam that can be phase conjugated by the crystal is strictly limited by the the product of its surface by its angular phase-matching range. For strong diffusion, this angular range is much smaller than that of the beam at the exit of the diffusing medium. To equalize these ranges, a microscope objective with a sufficient magnification must be inserted, as done in two recent experiments [19, 20]. However, as in these experiments, the transverse area of the diffused beam must be sufficiently small to remain after magnification not greater than the crystal transverse area. This is obtained by using a thinner medium and a first microscope objective to reduce the object size. Our new setup is described in fig. 6: we used identical $\mathrm{x} 20 ; \mathrm{NA}=0.35$ objectives. The first microscope (M1) makes a reduced image of the object on the sample (making better use of the shower-curtain effect), and the second objective (M2) makes an image of the sample at infinity. The high numerical aperture of the microscope objectives allows for a wide solid angle of light collection from the sample.

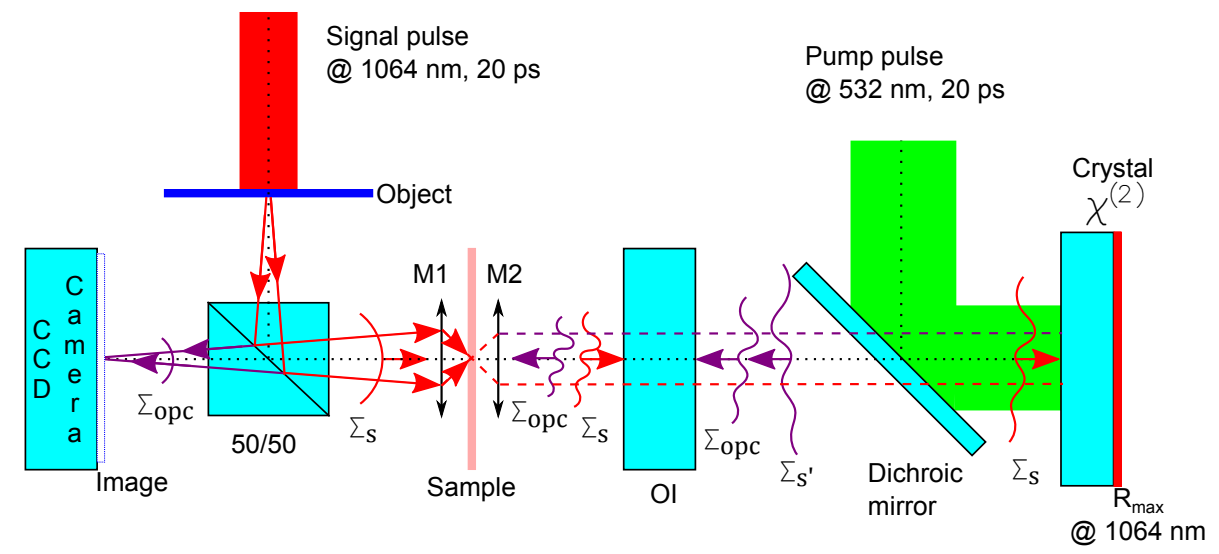

Figure 6. Phase-conjugation experimental setup with microscope objectives.

The rest of the setup is identical to the previous experiment: the scattered signal wave $\Sigma_{s}$ interferes with the pump pulse in the crystal, its reflection $\Sigma_{s^{\prime}}$ is rejected by the isolator OI and only the phase-conjugated wave $\Sigma_{o p c}$ is allowed through. As in the previous setup, the phase-conjugated-image is formed in the plane symmetrical to the object. We used a thin scattering sample similar to that used in $[9,10]$ : a microscope slab covered with a $0.05 \mathrm{~mm}$ layer of solid zinc oxide powder. With transmission measurements, we found that this layer corresponds to 7.4 mean scattering free paths.

The reflection from the sample cannot be avoided easily due to the real-time nature of our experiment, and here accumulating images does not help as the scattering medium 


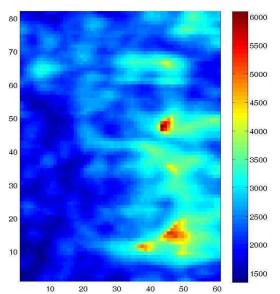

(a)

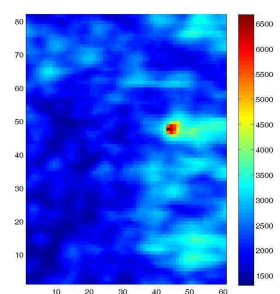

(b)

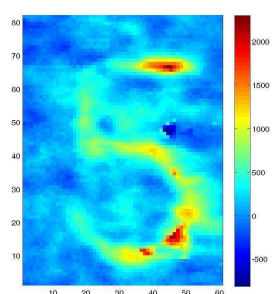

(c)

Figure 7. (a) Phase-conjugated image through the sample, (b) spurious reflected image obtained without pump (c) : subtracted image (a)-(b)

is static. Fortunately, the reflection occurs even in the absence of pump and can be subtracted of the phase-conjugated image. Fig.7(a) shows this phase-conjugated image of the fifth element of the zeroth group of the USAF resolution chart through the scattering sample. The spurious reflected image, obtained by blocking the pump, is shown in fig.7(b). By subtracting this spurious image from the phase-conjugated image, we obtain a clear improvement: fig.7(c). The maximum intercorrelation value of this image with the reference image of fig.9a (see below) is 0.61 .

\subsection{Etendue corresponding to phase conjugation}

Contrary to the first experiment where no imaging system was used, here M1 reduces the size of the object by 20 (the magnification of the microscope) on the sample. The scattering medium is considered to be thin enough so that the spot size at the exit face of the scattering medium is the same as the reduced image of the USAF resolution chart. The etendue E after the scattering medium is equal to the product of the spot size at the output face of the slab, $1410 \mu \mathrm{m}^{2}$, by the solid angle $\Omega^{\prime}$, still equal to $2 \pi$. The optical etendue after the scattering medium is now $E \sim 8.86 \times 10^{3} \mu \mathrm{m}^{2} . s r$. The etendue e collected by the microscope objective M2 is the product of the spot size at the output face of the slab, $1410 \mu \mathrm{m}^{2}$, by the solid angle $\Omega$ corresponding to the numerical aperture of the microscope objective $(\mathrm{NA}=0.35)$. We first verify that the beam in the crystal plane does not spread beyond the boundaries of the crystal and is almost completely phase-conjugated. The spot area in the crystal plane has been measured equal to 1.56 $m m^{2}$. The angle $\theta$ made by the light rays entering the crystal with the optical axis is given by the Abbe sine condition:

$$
\theta \approx \frac{N A}{\gamma}=17 \mathrm{mrad}
$$

where NA is the numerical aperture and $\gamma$ the optical magnification. $\theta$ is smaller than the maximum angle $\varphi$ allowed by phase matching (low gain limit): $\varphi=\sqrt{\lambda n_{s} /(2 L)}=19 \mathrm{mrad}$, where $\lambda$ is the signal and idler wavelength, $n_{s}$ the index of the crystal for the signal and $L$ the crystal length. For this value of $\varphi$, the crystal length is equal to the coherence length, i.e. the length for which the phase mismatch 
$\Delta K L$ is equal to $\pi[21]$. Therefore the etendue after M2 is equal to:

$$
e \sim 542 \mu m^{2} . s r \sim \frac{E}{6.010^{2}}
$$

Compared to the previous experiment the etendue ratio is 400 times superior.

\subsection{Comparison with single-pass imaging}

As in part 3, the phase-conjugated setup performances are compared to its single-pass equivalent as is shown in fig.8. Again, the isolator, dichroic mirror and KTP crystal are removed. The second objective no longer forms an image at infinity but at the usual imaging distance of microscope objectives $(160 \mathrm{~mm})$. The same object is imaged and shown first through a clean slab (fig.9a), then through the sample (fig.9b). The image is slightly deteriorated but remains visible with a maximum intercorrelation of 0.77 .

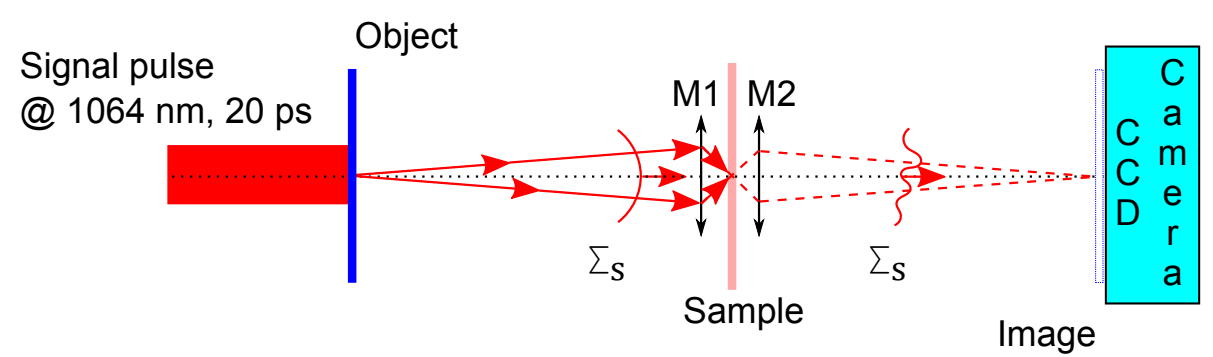

Figure 8. Single-pass imaging setup.

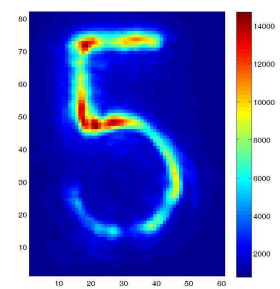

(a)

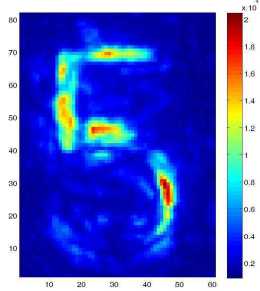

(b)

Figure 9. (a) Image through a clean microscope slab, (b) image through the scattering sample.

\section{Conclusion}

Phase conjugation by parametric amplification is extremely appealing because of its almost instantaneous character, allowing in principle imaging in in-vivo tissues, at a wavelength in the therapeutic window. However, this technique has also its drawbacks, in particular in terms of resolution related to phase-matching. We presented an ultrafast phase-conjugation imaging experiment through scattering media in the therapeutic window, first through a latex microbead solution then through a layer of zinc oxyde 
powder. We showed that we can form an image with phase conjugated light after back propagation in the diffusing medium. However, comparison with direct imaging does not show a clear improvement due do this back propagation. We will use in future research a thinner crystal, in order to increase the angular range where phase matching occurs and hence increase the phase conjugated etendue by using an optical system with a sufficiently high numerical aperture.

\section{Appendix}

We relate here the normalized intercorrelation coefficient to the image-to-background ratio in the detected image. The field $E_{d}$ at a point in the plane of the CCD camera can be expressed as [18]:

$$
E_{d}=\alpha E_{o}^{*}+\beta E_{n}^{*}
$$

Where $E_{o}$ is the true normalized object field and $E_{n}$ is a normalized noise field, generated because of the incomplete phase conjugation. This field is, in principle, orthogonal to the object field [18]. We will here more simply assume that this field is not correlated with the object field, ensuring a vanishing covariance between the corresponding intensities $I_{n}$ and $I_{o}$. The detected intensity $I_{d}$ is given by:

$$
I_{d}=|\alpha|^{2}\left|E_{o}\right|^{2}+|\beta|^{2}\left|E_{n}\right|^{2}+2|\alpha||\beta|\left|E_{o}\right|\left|E_{n}\right| \cos (\varphi)
$$

where $\varphi$ is a random phase, because of the absence of correlation.

The covariance between the normalized intensity $I_{o}=\left|E_{o}\right|^{2}$ in the original image and $I_{d}$ in the retrieved one becomes, with $I_{n}=\left|E_{n}\right|^{2}$ :

$$
\begin{aligned}
\left\langle I_{d}-\left\langle I_{d}\right\rangle\right\rangle\left\langle I_{o}-\left\langle I_{o}\right\rangle\right\rangle & =\left\langle I_{d} I_{o}\right\rangle-\left\langle I_{d}\right\rangle\left\langle I_{o}\right\rangle \\
& =|\alpha|^{2}\left\langle I_{o}^{2}\right\rangle+|\beta|^{2}\left\langle I_{n} I_{o}\right\rangle-|\alpha|^{2}\left\langle I_{o}\right\rangle^{2}-|\beta|^{2}\left\langle I_{o}\right\rangle\left\langle I_{n}\right\rangle \\
& =|\alpha|^{2} \operatorname{var}\left(I_{o}\right)+|\beta|^{2} \operatorname{cov}\left(I_{n}, I_{o}\right)=|\alpha|^{2} \operatorname{var}\left(I_{o}\right)
\end{aligned}
$$

While the last term in eq. 7 vanishes in the covariance, because it is not correlated with the other terms, it has to be taken into account in the computation of the variance of the detected image:

$$
\begin{aligned}
\operatorname{var}\left(I_{d}\right) & =|\alpha|^{4} \operatorname{var}\left(I_{o}\right)+|\beta|^{4} \operatorname{var}\left(I_{n}\right)+2|\alpha|^{2}|\beta|^{2}\left\langle I_{o}\right\rangle\left\langle I_{n}\right\rangle \\
& =\left(|\alpha|^{2}+\left.\beta\right|^{2}\right)^{2} \operatorname{var}\left(I_{o}\right)
\end{aligned}
$$

The last equality holds by assuming that the fields obey a thermal statistics law and because the fields are normalized. Hence the normalized coefficient $C$ reads:

$$
C=\frac{\operatorname{cov}\left(I_{d}, I_{o}\right)}{\sqrt{\operatorname{var}\left(I_{d}\right) \operatorname{var}\left(I_{o}\right)}}=\frac{|\alpha|^{2}}{|\alpha|^{2}+|\beta|^{2}} \Rightarrow \frac{C}{1-C}=\frac{|\alpha|^{2}}{|\beta|^{2}}
$$

To conclude, the normalized intercorrelation coefficient gives in principle access to the ratio of the powers of respectively the object intensity and the noise. However, in 
practice this coefficient appears as overestimated. Indeed, if the true covariance between the object image and the noise is zero, its estimation is non zero because we have defined $C$ as the maximum intercorrelation coefficient among all values obtained for different shifts between the images. While this shift can be due to a true shift between the object and the retrieved image, it can also be an artefact where the estimated covariance has a maximum due to the randomness of the data. This "random maximum" is as high as 0.2 for 300 independent data, scaling roughly as the inverse of the square root of the number of independent data. This number can be much lower than the number of pixels in the image because adjacent pixels are not independent. For example, the 0.47 intercorrelation of the image through water (fig.3a) with the signal (fig.3b) can be due in great part to this random part.

\section{Acknowledgments}

This work has been supported by the Agence nationale pour la recherche, project ANR2011-BSO4-017-03 "Imagerie Cohérente de Lumière Multidiffusée".

\section{References}

[1] D. Huang, E. A. Swanson, C. P. Lin, J. S. Schuman, W. G. Stinson, W. Chang, M. R. Hee, T. Flotte, K. Gregory, C. A. Puliafito, J. G. Fujimoto, "Optical Coherence Tomography," Science 254, 1178-1181 (1991).

[2] A.H. Gandjbakhche, " Diffuse optical imaging and spectroscopy in vivo ", C.R. Acad. Sci. Paris ser IV, 2 1073-1089, (2001), and references therein

[3] G. Le Tolguenec, F. Devaux, E. Lantz," Two-dimensional time-resolved direct imaging through thick biological tissues : a new step toward noninvasive medical imaging", Optics Letters 24 1047-1049 (1999)

[4] M. Atlan, B. C. Forget, F. Ramaz, A. C. Boccara, and M. Gross, "Pulsed acousto-optic imaging in dynamic scattering media with heterodyne parallel speckle detection", Optics Letters, 30 1360-1362 (2005)

[5] F. Helmchen ,W. Denk, "Deep tissue two-photon microscopy". Nat Methods 293240 (2005)

[6] E. N. Leith and J. Upatneiks, "Holographic imagery through diffusing media," J. Opt. Soc. Am. 254, 523 (1966).

[7] Z. Yaqoob, D. Psaltis, M. S. Feld, C. Yang, "Optical phase conjugation for turbidity suppression in biological samples," Nature Photon. 2, 110-115 (2008).

[8] N. Bazulev, N. Fomin, C. Fuentes, T. Hirano, E. Lavinskaya, S. Martemianov, T. Mizukaki, A. Nakagawa, S. Rubnikovich, J.-B. Saulnier, K. Takayama, and J.-L. Tuhault, "Laser monitor for soft and hard biotissue analysis using dynamic speckle photography," Laser Physics 13, 786-795 (2003).

[9] E. J. McDowell, M. Cui, I. M. Vellekoop, V. Senekerimyan, Z. Yaqoob and C. Yang, "Turbidity suppression from the ballistic to the diffusive regime in biological tissues using optical phase conjugation," J. Biomed. Opt. 15, (2010).

[10] F. Devaux, E. Guiot and E. Lantz, "Image restoration through aberrant media by optical phase conjugation in a type II three-wave mixing interaction," Opt. Lett. 23, 1597-1599 (1998).

[11] L. Landau and E. Lifchitz, "Electrodynamics of Continuous Media" Second Edition: Volume 8 (Course of Theoretical Physics), Elsevier (1984)

[12] M. Johnson, C. C. Mao, G. Moddel, M. A. Handschy and K. Arnett, " High-speed, low- 
power optical phase conjugation using a hybrid amorphous silicon/ferroelectric-liquid-crystal device" Opt. Lett. 20, 1114-1116 (1990).

[13] V.S.Sudarshanamand, M.Cronin-Golomb, P.R.Hemmer and M.S.Shahriar, "Turbulence aberration correction with high-speed high-gain optical phase conjugation in sodium vapor", Opt. Lett. 22, 1141-1143 (1997).

[14] D.C. Jones and K.D. Ridley, "experimental investigation by stimulated Brillouin scattering of incomplete phase conjugation", J. Opt. Soc. Am. B 14, 2657-2663

[15] F. Devaux, G. Le Tolguenec, E.Lantz, "Phase conjugate imaging by type II parametric amplification", Optics communications 147, 309-312 (1998)

[16] F. Devaux, E. Lantz, "Transfer function of spatial frequencies in parametric amplification : experimental analysis and application to picosecond spatial filtering", Optics Communications 114, 295-300 (1995)

[17] I. Dror, A. Sandrov and N. S. Kopeika, "Experimental investigation of the influence of the relative position of the scattering position of the scattering layer on image quality: the shower curtain effect," J. Opt. Soc. Am. 37, 6495-6499 (1998).

[18] C. Gu and P. Yeh, "Partial phase conjugation, fidelity and reciprocity", Optics Communications 107, 353-357 (1994).

[19] I. M. Vellekoop and A. P. Mosk, "Universal optimal transmission of light through disordered materials," Physical Review Letters 101, (2008).

[20] S. M. Popoff, G. Lerosey, R. Carminati, M. Fink, A. C. Boccara and S. Gigan, "Measuring the transmission matrix in optics: an approach to the study and control of light propagation in disordered media," Physical Review Letters 104, (2010).

[21] E. Lantz , F. Devaux, "Parametric amplification of images: from time gating to noiseless amplification", Journal of Sel. Topics in quantum Electronics 14, 635-647 (2008), eq. (3) 